García Miragall, Carlos.

Profesor Titular de Universidad, Universidad Politécnica de Valencia, Departamento de Sistemas Informáticos y Computación, Laboratorio de Luz.

Martínez de Pisón, María José.

Profesora Titular de Universidad, Universidad Politécnica de Valencia, Departamento de Pintura, Laboratorio de Luz.

Sanmartín Piquer, Francisco.

Profesor Titular de Universidad, Universidad Politécnica de Valencia, Departamento de Pintura, Laboratorio de Luz.

\title{
ANTIMATTER, un proyecto de audiovisualización en tiempo real de PDP11
}

\section{ANTIMATTER, a PDP11 real-time audiovisualization project}

TIPO DE TRABAJO: Comunicación.

PALABRAS CLAVE

Audiovisualización, Performance Audiovisual, Música Experimental, Improvisación, Tiempo Real.

KEY WORDS

Audiovisualization, Audiovisual Performance, Experimental Music, Improvisation, Real Time.

RESUMEN

El colectivo PDP11 es un grupo de artistas que provienen del punk, del rock, de la bossa nova, del cine experimental, del arte interactivo y del videoarte. En los inicios el colectivo centró su trabajo en las relaciones entre el sonido y la imagen. PDP11 construye su discurso visual al margen de lo tangible elevando el sonido al irracional firmamento de las matemáticas y desde allí, retoma impoluto a una nueva identidad, la dimensión de los colores. El sonido dejado al vaivén del momento toma su forma en ruidos, distorsiones, guitarras, sintetizadores, bajos, voces y silencios. Los proyectos se desarrollan de forma colectiva a partir de una idea modulada por las aportaciones personales durante su desarrollo. El proyecto Antimatter que se presenta está inspirado en el concepto físico de antimateria. El universo de las partículas y antipartículas, y sobre todo la transformación que se produce cuando colisionan fundamenta la estructura de este proyecto.

\section{ABSTRACT}

The PDP11 collective is a group of artists that come from punk, rock, bossa nova, experimental cinema, interactive art and video art. In the beginning, the collective focused its work on the relationships between sound and image. PDP11 builds its visual discourse on the margin of the tangible by raising the sound to the irrational firmament of mathematics and from there, it untaintedly takes on a new identity, the dimension of colors. The sound left to the swing of the moment takes its form in noises, distortions, guitars, synthesizers, basses, voices and silences. The projects are developed collectively from an idea modulated by personal contributions during its development. The Antimatter project that we present is inspired by the physical concept of antimatter. The universe of the particles and antiparticles, and especially the transformation that occurs when they collide, bases the structure of this project. 
García Miragall, Carlos; Martínez de Pisón, María José; Sanmartín Piquer, Francisco

ANTIMATTER, un proyecto de audiovisualización en tiempo real de PDP11

IV Congreso INTERNACIONAL DE INVESTIGACIÓN EN ARTES VISUALES ANIAV 2019 IMAGEN [N] VISIBLE]

http://dx.doi.org/10.4995/ANIAV.2019.9057

\section{INTRODUCCIÓN}

Este trabajo forma parte de los resultados del proyecto de investigación subvencionado por la Conselleria d' Educació, Investigació, Cultura i Esport de la Generalitat Valenciana, proyecto GV/2017/28 titulado: Cíclope, visualización creativa de sonido basada en propiedades perceptuales del sonido aplicada a la realización de eventos audiovisuales en directo. Primero se presenta un resumen del colectivo PDP11, luego la forma con la que el colectivo aborda sus proyectos y finalmente se detalla el proyecto Antimatter que tuvo lugar en el Mattatoio de Roma dentro del festival Live Performer Meeting del 2018.

\section{EL COLECTIVO PDP11}

"Como prolongados ecos que de lejos se confunden en una tenebrosa y profunda unidad, vasta como la noche y como la claridad, los perfumes, los colores y los sonidos se responden." Charles Baudelaire.

PDP11, nació con la finalidad de establecer una plataforma abierta a la investigación del sonido y la imagen en su lado más cercano a la tecnología digital en el año 11. El nombre PDP11 es un homenaje a uno de los primeros miniordenadores de la empresa Digital, Programmed Data Processor (PDP versión 11), en cuyo nombre intencionadamente no se quería que apareciera la palabra computer debido a que en los años 70 se asociaba con un aparato muy voluminoso. En la serie 11 por primera vez se interconectan todos los elementos del sistema: procesador, memoria y periféricos, en un espacio reducido, plasmando la concepción actual que tenemos de los ordenadores personales. Esa idea de reunión de diferentes elementos es la que toma como referencia del colectivo.

El colectivo PDP11 es un grupo de artistas que provienen del punk, del rock, de la bossa nova, del cine experimental, de las artes visuales, del videoarte y de la programación de ordenadores entre otras disciplinas (ver figura1). A lo largo de los años ha habido personas que han colaborado puntualmente en un proyecto y otras que han participado en varios e incluso en todos.

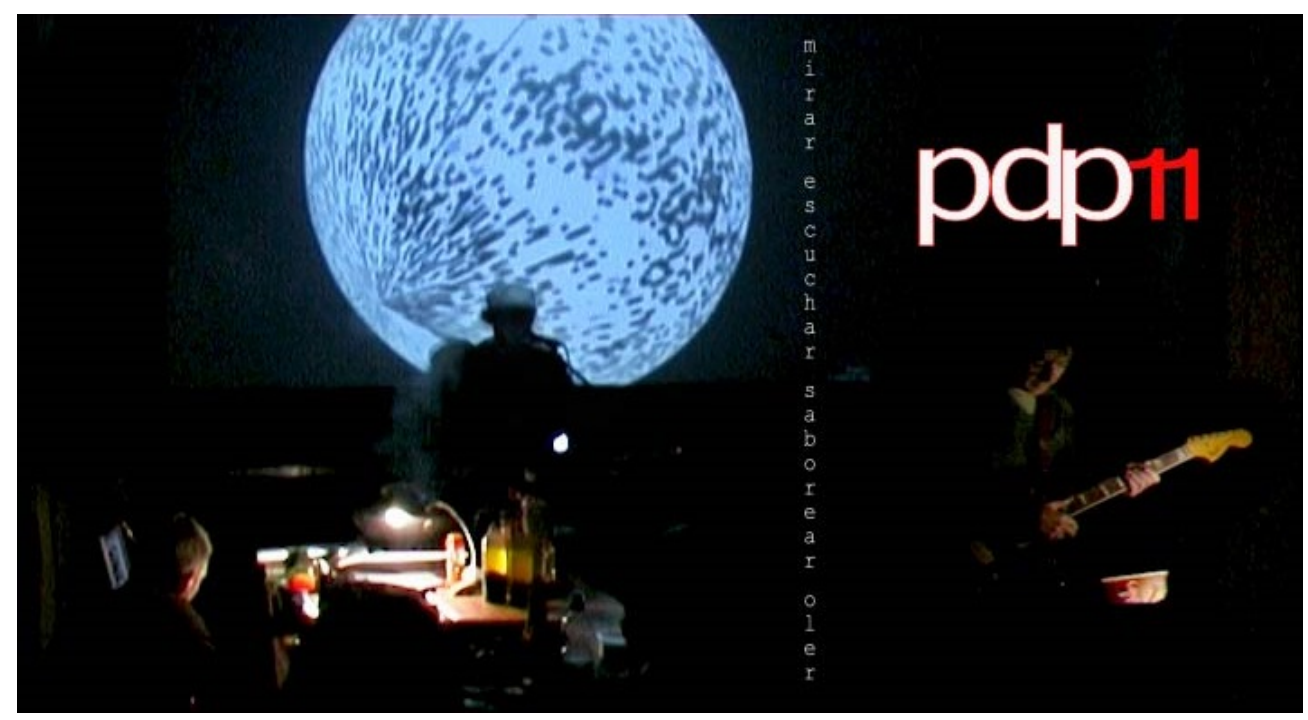

Figura 1. Cartel oficial de los inicios de PDP11. (Fuente: Carlos G. Miragall, 2012).

Los intereses principales de investigación son: Arte Sonoro, Arte Visual, Arte Culinario y Arte Multisensorial. La actividad del colectivo se plantea en formato de proyectos abiertos. En cada uno de los proyectos se define un modelo conceptual sobre el que se estructura su desarrollo. Cada uno de los participantes aporta sus ideas y en función de la disponibilidad personal y las características del espacio y audiencia se adecua el proyecto, dejando siempre un espacio abierto a la improvisación.

"La idea desde el principio fue hacer música libre de ataduras académicas y costumbristas. La música debía surgir espontánea y viva aferrándose únicamente a pautas alejadas de nociones clásicas. La imagen se concebía como una representación de la música, buscaría el color del sonido, con una mirada a veces detallista y otras confusa y borrosa. Todo ocurría en el momento, naciendo y muriendo al vaivén del tiempo que imparable desdibujaría su paso en el futuro. Bajo estas premisas se gesta en el año 11, el colectivo PDP11" (Laboratorio de Creaciones Intermedia, 2018). 
Como grupo interdisciplinar las influencias provienen de múltiples campos del conocimiento. El movimiento Fluxus es quizá uno de los referentes que mejor recoge la filosofía del grupo, en palabras de George Maciunas: "el arte diversión debe ser simple, divertido, no pretencioso, preocupado por las insignificancias, que no requiera habilidades o ensayos interminables, que no tenga valor ni institucional ni como mercancía" (1965). Las ideas que pautaron los futuristas en su manifiesto sonoro del arte de los ruidos, donde la música se expandía acogiendo a todos los sonidos del mundo (Russolo, 1913), y la actitud experimental del Video Arte han constituido otros de los cimientos del devenir de PDP11.

El sonido como guía para el desarrollo de imágenes que abanderó la Música Visual ha marcado desde el comienzo la línea visual de PDP11. El artista Norman McLaren es quizá uno de los mejores representantes de esta forma de proceder, cuyas propuestas fueron desde el trazado a mano del sonido hasta la generación automática de la imagen en función del sonido (Baquedano, 1987). Por otro lado el trabajo de la cineasta Mary Ellen Bute cuyo discurso visual tomaba como referencia la visualización técnica del sonido mediante un osciloscopio trasladándolo al campo de las artes, constituye un referente fundamental del colectivo.

La actitud disruptiva que el movimiento Punk adoptó como seña de identidad a finales de los sesenta (Strongman, 2008), y el otro lado de rock donde se fusionan sus estructuras sencillas con la complejidad conceptual de la Música Experimental (Carrera, 2014), también han influido en la forma de hacer de muchos de los proyectos del colectivo.

\section{LOS PROYECTOS DE PDP11}

PDP1 desarrolla su actividad en pequeños proyectos multisensoriales e interdisciplinares, combinado luz, sonido, olores o sabores. Los proyectos tienen un formato de performance en tiempo real. Todo parte de una idea que propone uno o varias personas del colectivo, en ocasiones influenciada por la temática del festival, el espacio de realización o las inquietudes personales y a partir de aquí se realiza un proceso de investigación que concluye en la definición de un marco referencial y una estructura base para el desarrollo de la performance. Las partituras son guiones literarios, recetas de cocina, esbozos de sensaciones o teorías físicas y matemáticas dejando los detalles al devenir del momento, al espacio o a la interacción con el público. Las propuestas del colectivo han ido desde temas comprometidos como la prostitución hasta procesos sencillos como la elaboración de una receta de cocina. En la figura 2 se puede ver una recopilación de los carteles de los diferentes proyectos que ha desarrollado el colectivo.
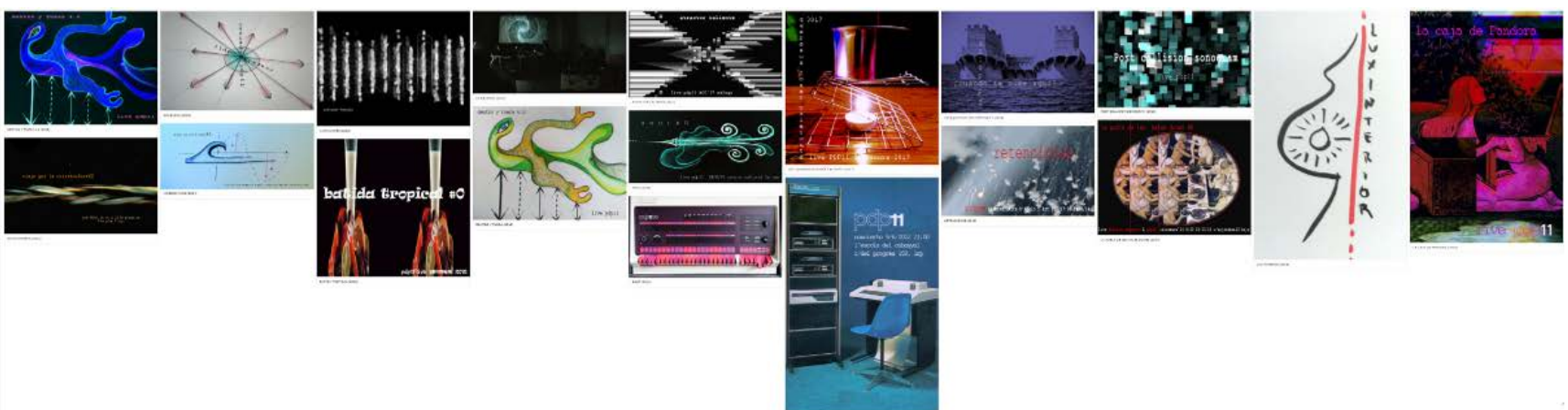

Figura 2. Carteles de diferentes proyectos de PDP11. (Fuente: www.pdp11.es).

Desde el punto de vista sonoro se ha trabajado con guitarras y bajos eléctricos procesados, sintetizadores, máquinas de escribir, voces, sonidos encontrados o grabaciones realizadas durante la propia performance entre otros recursos. El visual además de la partitura general siempre toma como base el sonido que en ese momento se está generando. Se han desarrollado y utilizado aplicaciones como Ciclope que generan automáticamente visuales a partir del sonido, traduciendo matemáticamente muestras de sonido a pixeles (García \& Sanmartín, 2017) o GAmuza que establece un entorno de trabajo para diseñar visuales usando las librerías de OpenFrameworks (Martínez de Pisón \& Mazza, 2016).

En algunos proyectos como "La caja de pandora", "Queremos comer caliente" o "Batida tropial", el colectivo ha introducido sabores y olores en la realización de las performances buscando una inmersión y participación de las personas asistentes más directa y tratando de abordar una experiencia multisensorial completa. 
García Miragall, Carlos; Martínez de Pisón, María José; Sanmartín Piquer, Francisco

ANTIMATTER, un proyecto de audiovisualización en tiempo real de PDP11

IV Congreso INTERNACIONAL DE INVESTIGACIÓN EN ARTES VISUALES ANIAV 2019 IMAGEN [N] VISIBLE]

http://dx.doi.org/10.4995/ANIAV.2019.9057

Una revisión detallada de todos los proyectos que ha realizado el colectivo se puede encontrar en su página web: www.pdp11.es (consultado 18 de marzo 2019).

\section{ANTIMATTER}

Antimatter es un proyecto inspirado en el concepto físico de antimateria (ver figura 3). El universo de las partículas, antipartículas y la transformación que se produce cuando colisionan han fundamentado la estructura de este trabajo. Este proyecto se presentó en Mattatori de Roma dentro del festival Live Performer Meeting en Mayo de 2018 y participaron María José Martínez de Pisón con los visuales, Carlos García Miragall con guitarra eléctrica y efectos y Francisco Sanmartín con sintetizadores, con una duración de 25 minutos.

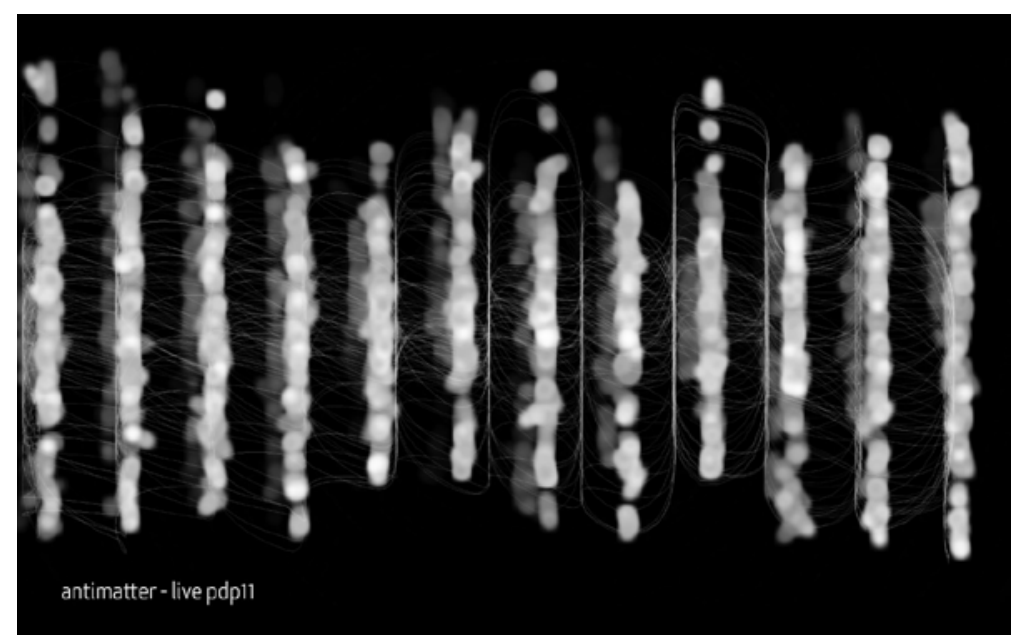

Figura 3.Cartel genérico del proyecto Antimatter. (Fuente: María José Martínez de Pisón, 2018).

La teoría física sobre la antimateria establece que junto con las partículas existen sus reversos, las antipartículas. Este descubrimiento es debido a Paul Dirac cuando trataba de unificar las teorías de la relatividad y la mecánica cuántica, formulando la llamada ecuación de Dirac: $(\partial+m) \psi=0$ (Berry, 1998). A partir de este momento los científicos descubrieron los antielectrones o positrones, los antiprotones y los antineutrones, así como los enigmas de su comportamiento. Uno de los aspectos más interesantes es que cuando una partícula colisiona con su correspondiente antipartícula, se aniquilan generándose el proceso más energético que existe en el universo. Para una descripción detallada sobre la antimateria se puede consultar (Gato, 2018)

Antimatter toma como marco conceptual la idea de los contrarios y cómo su encuentro produce una de las mayores fuentes de energía del universo para terminar con su aniquilación. Bajo estas premisas se desarrolló la estructura de la performance audiovisual. en tres partes. En la primera parte se planteó un intercambio entre opuestos, alternado las partículas con sus contrarios, en la segunda parte se producía un acercamiento paulatino entre las partes enfrentadas y finalmente se generaba la colisión desdibujando la materia de la antimateria.

\section{Visualización}

Es posible representar cuantitativamente el tiempo y los valores de las frecuencias de la señal de audio utilizando un oscilógrafo, un espectrógrafo de sonido, o algoritmos que sustraen digitalmente sus datos a través del análisis del volumen, el pitch, la FFT o las bandas de Mel. Otras visualizaciones se derivan de representaciones de la música basadas en notas o partituras, transferidos a eventos de notas MIDI (Smith \& Williams, 1997; Sapp, 2001). Todos ellos suponen sistemas de parametrización del audio; pero a la hora de representar esos valores (esos vectores o arrays de números que provienen de señales de audio polifónicas) en un espacio visual bi o tri-dimensional es necesario transferir los datos cuantitativos a composiciones cualitativas. Esa transferencia implica el uso recursos estéticos para que la información se pueda asignar, por ejemplo, a las dimensiones espaciales, al movimiento de las formas, 0 al color. 
En un segundo nivel hay otros factores que incidirán más en la expresión estética de esas configuraciones, por ejemplo la borrosidad o nitidez de las formas, o sus niveles de contraste, y es en estas valoraciones donde la visualización del sonido se aproxima a las cualidades del video-arte o el cine experimental, entendiéndolas como signos sensomotores - cuando se establece una relación más directa del sonido con la sensación de movimiento y acción- o, como señala Deleuze $(1987$, p. 25) como "opsignos" y "sonsignos" (signos ópticos y sonoros), porque el poder descriptivo de formas, colores y sonidos reemplaza y recrea de manera cualitativa el propio objeto representado. Entonces, el movimiento de las formas y colores, impulsado por los datos del sonido, puede pasar a ser vagabundeo, flotación, viaje por el espacio o confrontación de materia y antimateria. Metáforas que articulan las relaciones de lo sonoro y lo visual y que se configuran por el encuentro de la aprehensión objetiva de los datos cuantitativos con la fantasía e imaginación cualitativa de la improvisación sonora y la composición visual.

En una duración temporal casi imperceptible (que denominamos tiempo-real) se da la improvisación sonora, a partir de ella se hace el cálculo técnico de los datos sonoros, y después, la adaptación de estos datos en la configuración visual. Esto implica el encuentro de la intuición con las formulaciones matemáticas, un encuentro modulado por estructuras y diagramas que articulan el desarrollo del código a través de lenguajes de programación creativa. Para este proyecto se utilizó el software GAmuza, un entorno de scripting hecho con openFrameworks (OF), inspirado en Processing, uniendo el lenguaje de OF 0.8.4, el de OpenGL 1.1, y un pequeño framework propio de GAmuza con funciones y módulos GUI para hacer la programación creativa mucho más sencilla, todo ello embebido en un entorno de programación de lenguaje Lua ligeramente modificado (Martínez de Pisón \& Mazza, 2016).

La estructura de programación se articulaba en diferentes estados; en el primero, los datos del sonido impulsaban a los elementos de la composición en forma de torbellino; en el segundo, modulaban fluidos lineales al incorporarse en funciones noise; en el tercero tomaban como referencia un ejemplo de Daniel Shiffman para un sistema de partículas (Shiffman, 2012) con texturas de imagen y mezcla aditiva que aportaba a la imagen la indefinición y borrosidad propias del humo. En cada uno de estos estados el flujo sonoro se reflejaba en el comportamiento dinámico de las formas y la luz, escalando su tamaño con cada golpe sonoro, provocando giros y traslaciones, como bloques de movimiento que reflejaban el flujo de elementos indefinidos o desdibujados, el encuentro ente partículas que metafóricamente aludían a la idea de antimateria.

\section{Sonido}

El discurso sonoro se plantea mediante dos instrumentos, una guitarra eléctrica y un sintetizador modular. Durante el desarrollo de la performace la guitarra adopta el rol de la materia y el sintentizador el de la antimateria.

La materia y sus partículas se definen armónicas y claras, con sonidos afinados que alternan en graves y agudos. El ritmo acompasa sus movimientos buscando una mirada de lo real, de lo conocido, de lo tangible y observable. La antimateria es obscura, es ruidosa y permanece en un segundo plano, como los sonidos indeseables que la mente humana rechaza en un intento de anular sus efectos.

El acercamiento de los opuestos se produce cuando el universo claro y limpio de la materia se torna impreciso y distorsionado. El ritmo se aleja y la imprecisión toma su lugar. La antimateria oculta pero latente, poco a poco gana importancia mostrándose más precisa y más cercana a la materia, su estructura no ha cambiado, pero ahora las defensas mentales han dejado de ser efectivas. Finalmente, la colisión produce un acercamiento ya casi mimético donde los opuestos se han desdibujado que concluye con su aniquilación.

A nivel técnico se ha usado una guitarra eléctrica modelo Fender Jazzmaster procesada con pedal efectos de Distorsión de la empresa Boss, un Delay estéreo también de Boss y un Looper estéreo de la marca Ditto. Los sintetizadores usados han sido tres Moog Mother 32 interconectados entre sí.

\section{CONCLUSIONES}

En este trabajo hemos presentado el devenir del colectivo PDP11, sus orígenes, sus influencias y su forma de proceder en el desarrollo de proyectos, siempre marcada por la búsqueda de relaciones entre la imagen y el sonido. Antimmater es una reflexión sobre la importancia de los contrarios y sobre sus colisiones, metafóricamente representada en una performance audiovisual desarrollada en tiempo real para el festival Live Performer Meeting, un encuentro entre artistas multidisciplinares propenso al intercambio de ideas y tecnologías. Debido a la gran cantidad de propuestas que se presentaron, la organización reservó muy poco tiempo para la realización de las pruebas de sonido e imagen y algunos proyectos como el de PDP11 que requería de una preparación más compleja se vieron perjudicados, teniendo que dar soluciones apresuras a problemas técnicos. Esto es habitual en las artes vivas y debe tenerse en cuenta siempre con la finalidad de evitar una realización imprecisa y desmotivada.

De nuevo y focalizando el interés en las relaciones entre el sonido y la imagen se buscó en Antimatter una mirada íntimamente seducida por el sonido buscando como en casi todos los proyectos bailar con el color de la música. 
García Miragall, Carlos; Martínez de Pisón, María José; Sanmartín Piquer, Francisco

ANTIMATTER, un proyecto de audiovisualización en tiempo real de PDP11

IV Congreso INTERNACIONAL DE INVESTIGACIÓN EN ARTES VISUALES ANIAV 2019 IMAGEN [N] VISIBLE]

http://dx.doi.org/10.4995/ANIAV.2019.9057

\section{FUENTES REFERENCIALES}

Bakedano, J. (1987). Norman McLaren. Obra completa 1932-1985. España: Museo de Bellas Artes de Bilbao.

Berry, M. (1998). Paul Dirac: The purest soul. Physics World, (11), 36-40.

Deleuze, G. (1987). La imagen-tiempo: estudios sobre cine 2. Barcelona: Paidós.

Carrera Sánchez, O. (2014). Malas hierbas: historia del rock experimental (1959-1979). Madrid, España: T\&B.

García Miragall, C.M. y Sanmartin Piquer, F. (2016). De números a números: Visualización del sonido por métodos relacionales de muestreo en directo. AusArt Journal for Research in Art, 4 (1), 105-117.

Gato Rivera, B. (2018). Antimateria. Madrid, España: Consejo Superior de Investigaciones Científicas.

Laboratorio de Creaciones Intermedia (ed.). (2018). Protoperformance en España (1834-1964). Lucena-Córdoba, España: Weekend Proms.

Martínez de Pisón, M. J., y Mazza, E. (2016). Live Creative Coding. Introducción a la programación creativa con GAmuza. Valencia: Plutón Asociación cultural.

Maciunas, G. (1965). Manifesto on Art / Fluxus Art Amusement.

Russolo, L. (1913). El arte de los ruidos. Carta dirigida a Francesco Balilla Pratella.

Sapp, C. S. (2001). Harmonic Visualizations of Tonal Music. Presentado en International Computer Music Conference, San Francisco, California.

Shiffman, D. (2012). The nature of code. United States: D. Shiffman.

Smith, S., y Williams, G. (1997). A Visualization of Music. Presentado en Visualization '97. New York: Association for Computing Machinery.

Strongman, Phil. (2008). Historia del punk: El movimiento juvenil que transformó la escena musical y social en el mundo. Barcelona, España: Robinbook. 